\title{
APPLIANCES
}

\section{A NEW ELECTRONIC TONOMETER*}

\author{
BY \\ J. FRANÇOIS, R. MOENS, and R. MOENS \\ From the Ophthalmological Clinic and the Physics Laboratory, \\ University of Ghent, Belgium
}

A tonometer must possess three essential properties:

(a) It must be very sensitive in order to give the ocular tension with exact precision. This has not hitherto been achieved by any other mechanical process.

(b) It must measure the tension without taking into account the resistance and elasticity of the globe, two factors which vary from eye to eye and constitute an obvious source of error which must be eliminated.

(c) It must work in a simple way to make it practicable for clinical use.

With these principles in mind we produced the electronic tonometer which we now present (Fig. 1).

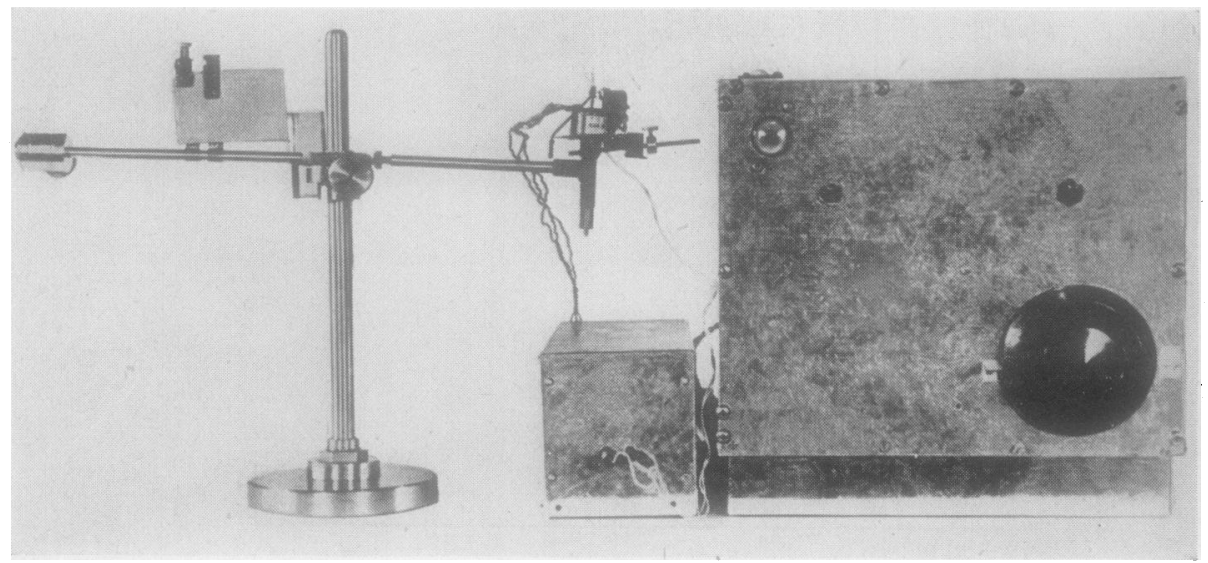

FIG. 1. Electronic tonometer (general view).

\section{Construction of Apparatus}

The apparatus (Fig. 2) consists of a small box (1) with an open tube (2) at its base. The tube is $5 \mathrm{~cm}$. long with an opening $8 \mathrm{~mm}$. in diameter. The box is held by a horizontal arm (3), pivoted at its centre (4) to a rack-like device (5), which permits the apparatus to move up and down a vertical column (6).

The box and arm are balanced by a counterweight (7) and fixed in the horizontal position by means of a weak spring (8), which eliminates the danger of contusion or injury to the globe, should the patient move unexpectedly.

Inside the tube (2), but without touching the sides, slides a very light rod (9),

- Received for publication June 14, 1952. 


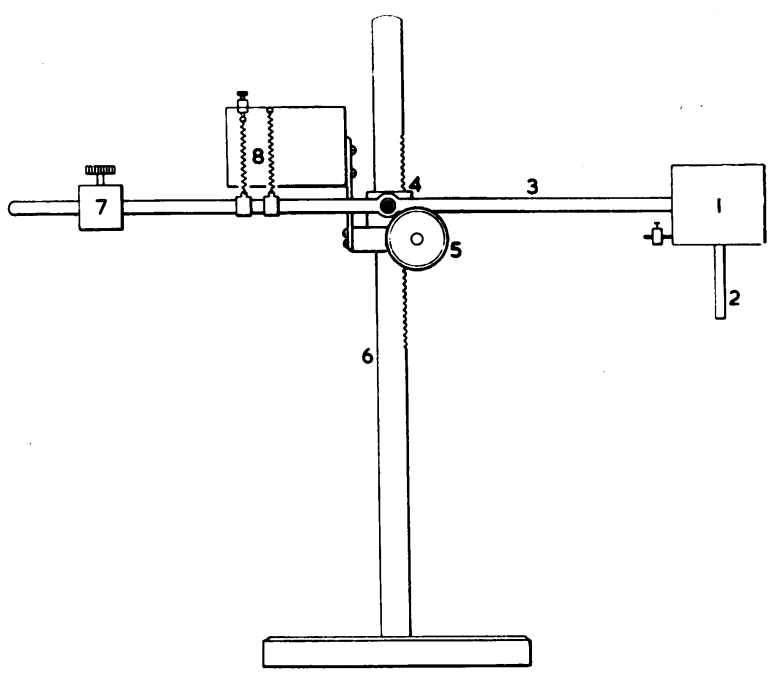

$3 \mathrm{~mm}$. in diameter (Fig. 3). This rod is supported by a small horizontal arm (10), which is articulated on a pivot (11) and balanced by a counterweight (12). The counterweight must be so adjusted that the rod (9) may be made to rest very lightly upon the cornea, when the surface of the cornea is brought into contact with the end of the tube (2). In this position of rest $(0 \ldots . .0)$ the end of the rod (9) is found at a distance $x_{0}$ from the line $0 \ldots 0$.

A weight of $7.5 \mathrm{~g} .(13)$ is retained above the arm (10) by a small

Fig. 2. Diagram of apparatus, showing tube (2) designed to rest upon the cornea, and counterweight (7).

electromagnet 14); if the current to the magnet is cut, the weight (13) falls upon the arm (10), and the end of the rod (9) descends to a new level $x_{1}$.

\section{Method of Application}

The distance from $x_{1}$ to $x_{0}$ gives us the exact degree of the ocular tension, and it is possible to measure this distance with the greatest precision by electronic methods. For this purpose we fix to the top of the rod (8) a small steel armature (15). Any movement of this armature will vary the self-induction caused by the magnet (16) on the bobbin (17). For every position of the rod (9)-that is, for every value of $x$-there is a corresponding value of the self-induction. As this selfinduction sets off an oscillating circuit of an oscillating triode, this value corresponds to a frequency $F$ (audible frequency).

We have constructed a second oscillator which includes an identical bobbin, the 
armature of which may be displaced at will by means of a micrometer screw fixed to a graduated dial (the screw has a thread of $0.5 \mathrm{~mm}$., and the dial is graduated into 200 divisions).

It is now possible, by a measured displacement on the dial of the micrometer screw, to set this armature in a position identical with that of the first oscillator, thus making the frequency of the second oscillator the same as that of the first $(F)$.

This equality of frequency may be obtained and determined precisely by means of the acoustic beats produced by the two oscillators. These beats. can also be shown by means of a visual measuring apparatus (the "magic eye").

Thus, for each position of the rod (9) an indication on the dial of the micrometer screw enables $x$ to be measured exactly.

As a measure of control the apparatus has been graduated for a series of values $x\left(x_{1}, x_{2}, x_{3}\right.$, etc.), varying from $-2 \mathrm{~mm}$. to $+2 \mathrm{~mm}$., and the corresponding graduation for each of these values of $x$ has been determined and indicated on the dial of the micrometer screw. These values of $x$ were determined by placing under the rod small copper disks of different thicknesses (measured exactly by a spherometer). By noting the reading on the micrometer dial which corresponds to a copper disk of given thickness, a linear graph (Fig. 4) was obtained, which

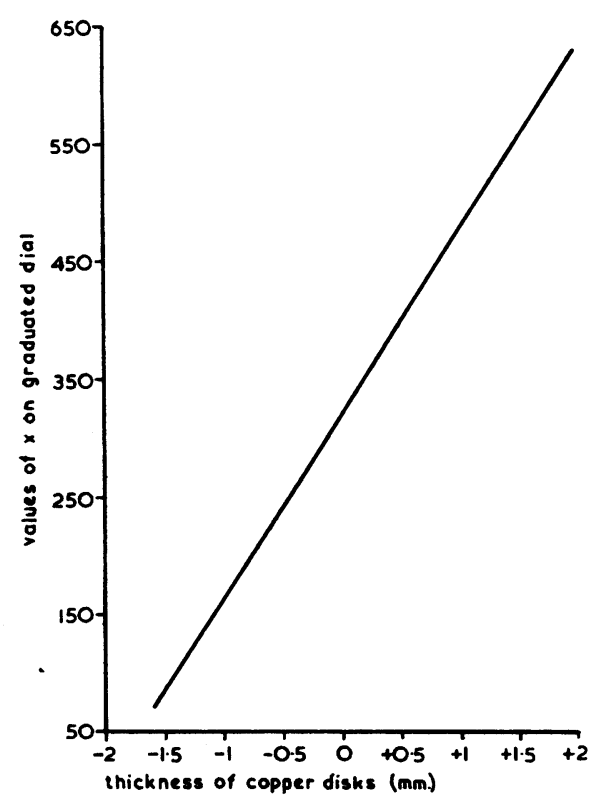

Fig. 4. Graph to show value of $x$ corresponding with displacements of rod (9). shows the measurement of ocular tension exactly corresponding to each given displacement of the rod.

\section{Clinical Procedure}

The patient lies flat, with his head horizontal, and gazes in an exactly vertical direction. The cornea is anaesthetized with pantocaine. The apparatus is then lowered down the rack and adjusted by means of the counterweight (7) until the end of the tube (2) is poised above the eye. The rod (9) is adjusted by means of the counterweight (12) until it rests lightly upon the surface of the cornea. While these adjustments are made the weight (13) must be held up by the electromagnet. The position of the micrometer screw which gives the second oscillator the same frequency as that of the first is observed, and the graduation on the dial is noted. The magnetic current is now interrupted; the weight (13) falls and bears upon the arm (10), which in its turn depresses the rod (9). The new position of the micrometer screw, which is now necessary to equalize the frequency of the two oscillators, is observed, and the new graduation on the indicator dial is noted. The difference between the first reading and the second gives the degree of ocular tension. 


\section{Results}

To standardize and test the apparatus we determined the ocular tension in a large number of people, first with the Schiötz tonometer and then with our own. In these tests, the difference between the two figures indicated by the micrometer screw of the electronic tonometer was found to be in inverse proportion to the number of millimetres of mercury measured by the Schiötz apparatus. The various values followed a linear curve, which increased gradually for the higher tensions (Fig. 5).

It is evident that to standardize our apparatus precisely it would be necessary to use an intra-ocular manometer, and this we propose to do ultimately. A comparison of the figures given by our apparatus with those given by the Schiötz tonometer does in fact introduce an element of inaccuracy, since the Schiötz readings are necessarily influenced by the elasticity and resistance of the globe.

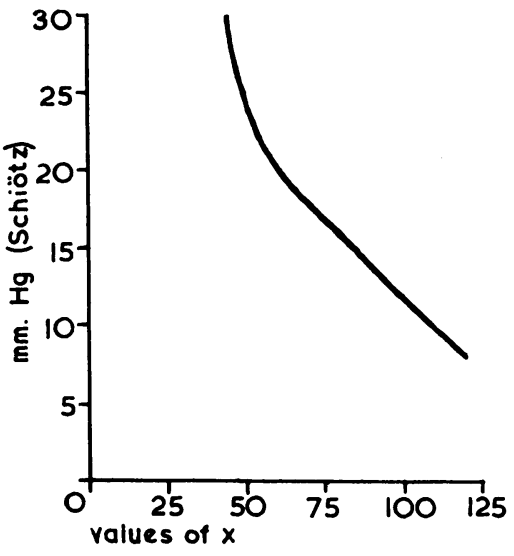

FIG. 5. Graph to show values of $x$ corresponding with measurements obtained by Schiötz tonometer. 